\title{
Resource verification of construction objects having increased importance level
}

\author{
Nadezhda Sevryugina* and Pavel Kapyrin \\ Moscow State University of Civil Engineering, Yaroslavskoe shosse, 26, Moscow, 129337, Russia
}

\begin{abstract}
It has been established that scientific notations in common take construction infrastructure as a complicated system capable for selfdevelopment and process self-regulation. It is proposed to use variative verification modelling process approach for reliability substantiation of construction objects having increased importance level. "Safety" indicator is accentuated as the principal one among other complex reliability indicators. Alternative algorithm for safety level smart control was presented. The algorithm includes scientific and technical rating of hazardous situations, and their parametric standardization for objects increased importance level. Graphical visualization of hazardous situation occurrence risk probability under varying shift of reliability parameter during operation period was obtained. The inverse problem was also solved: restrictions on controlled parameters shift values are introduced for specified reliability level and set hazard occurrence probability.
\end{abstract}

\section{Theoretical and practical prerequisites}

Review of principal theories and methods shows that scientific researches emphasize reliability, energy efficiency and safety problem of construction objects having various importance levels. In particular, research results in papers [1-8] are methods providing theoretical characteristics of urban development and city development regulation. Common feature of all these methods is construction infrastructure representation as a complicated system capable for self-development and process self-regulation.

The analysis shows that requirements on construction object reliability conform with generally accepted requirements in adjacent economy spheres, in particular, limit state is registered under critical load combination during design operation period. However, it should be mentioned that results achieved in time period vary considerably as the recommended life time of unique buildings and structures is 100 years and longer [9-11].

Study purpose: substantiate implementation of resource verification system for construction objects having increased importance level by means of multi-criteria reliability rating with "safety" as the dominant parameter during operating stage of the object's lifecycle.

\footnotetext{
* Corresponding author: SevryugiNS@ mgsu.ru
} 


\section{FMEA standard}

FMEA requirements are efficient for relevant resources provision. At the same time complete information on features and requirements to the object as a system is required.

Detailed FMEA developed for a specific system can be updated and improved for subsequent system modification in case of full conformity of structure, load, and other parameters. If differences in external condition and operating load are found out, new FMEA will be required.

Information on functions, features and parameters is required at all system levels. System borders are set. The borders include physical and functional interface of the system and its environment, including other systems with which the system interacts. The standard presumes that principal rules of system levels selection for analysis depend on the desired result and availability of required information.

The level characterized by available information required to determine and describe its functions based on primary previously stated reliability, maintainability and safety level, is taken as the lowest system level, which allows to lower analysis specification level. Consequently, we should put emphasis on historical data on reliability, maintainability and safety of identical systems.

FMEA results are taken as a base for strategy development of safe operation of construction objects having increased importance level (COIIL), but initial database should be corrected under real-life situations. Data bank of system variations in time should be created. In a timeframe, FMEA failure probability estimate relates to resource value or specified object lifetime.

Risk assessment is a number if logical steps providing systematic hazards examination. Functional $F$ linking probability $P$ of undesired event and mathematical expectation of loss $U$ resulted from this undesired event [risks] is a base for risk assessment $R$ under technical regulation:

$$
R=F_{R}\{U, P\}=\sum_{i}\left[F_{R}\left(U_{i}, P_{i}\right)\right]=\int C(U) P(U) d U=\int C(P) U(P) d P,
$$

where $i$ - types of undesired events,

$C$ - weight functions taking account of risks interaction.

The following value is used in some FMECA types as a general measure of potential risk $R$ :

$$
R=S \times P,
$$

where: $S$ - value of severity of consequences, i.e. value of failure effect on a system or a user (non-dimensional value);

$P$ - failure detection probability (non-dimensional value).

In some applications FMEA or FMECA can additionally mark failure detection level for the system as a whole. In this case additional failure detection value $D$ is used (which is also a non-dimensional value) to obtain risk priority value $R P N$ :

$$
R P N=S \times O \times D,
$$

where: $O$ - failure probability for a set or specified time period (this value can be described as a rank instead of actual failure probability value);

$D$ - specifies failure detection and assesses the chance to identify and eliminate failure before after-effects for the system or the user. $D$ values are usually ranked in reverse order in respect of failure probability or failure severity. The higher $D$ value is, the lower 
failure detect ion probability is. Lower failure detection value corresponds to higher RPN value and higher failure type priority.

It is worth mentioning that it is recommended to use Probabilistic Risk Analysis (PRA) instead of FMECA for systems with high risk or high construction complexity.

Common Cause Failure (CCF) examination includes "human factor" parameter, as many $\mathrm{CCF}$ failures are caused by human errors. Software errors are separated on the grounds that FMEA-based solutions on consequences, criticality and conditional probability can depend on software elements, their special features, sequence and time of operation.

For COIIL safety valuation it is necessary to analyze principal groups of affecting factors. The analysis is based on critical $\left(E_{c}, W_{c}, I_{c}\right)$ and maximum allowable characteristic properties $([E],[W],[I])$ of human, technosphere and environment resistance to these factors (including assignment of maximum allowable concentration $[d E / d F],[d W / d F]$, doses $[(d E / d F) d t],[(d W / d F) d t],[d I / d t]$, vulnerability and damage levels).

Probabilistic modeling and integration (summation) is carried out for every combination of factors affecting on COIIL engineering systems and structures, and their maximum allowable values.

The modeling considers function of distribution in area $F$ and time $t$ to specify risks $R$, damage $(D)$ or vulnerability $V$ of a man $N$, technosphere objects $T$ and environment $S$ by means of actual-to-critical values ratio of hazardous energy, substances, information flows (or its concentration and dose) [risks]:

$$
\left\{D_{r, t}, V_{F, t}\right\}_{F, t}=F_{D, V}\left\{\left(\frac{E}{E_{c}}\right),\left(\frac{W}{W_{c}}\right),\left(\frac{I}{I_{c}}\right)\right\}=\iint_{F t}\left[\left(\frac{E}{E_{c}}\right),\left(\frac{W}{W_{c}}\right),\left(\frac{I}{I_{c}}\right)\right] \frac{d F}{d t}
$$

Loss values UF,t are assessed on the basis of determined damage $D F, t$ and vulnerability $V F, t$ values for specified probability values $P F, t$.

According to basic theory, calculations allow to determine risk values $R F, t$ for a given point $F$ and time $t$, and prepare a risk map.

Scientifically grounded maximum allowable levels allow to report the safety provision in the following form:

$$
\left\{R, R_{F, t}\right\} \leq\left\{R|,| R_{F, t} \mid\right\}
$$

Comprehensive methods and their modifications are used for risk analysis in complicated systems.

In addition to high-quality methods realizing formal hazards and consequences evaluation procedures it is necessary to develop affection area (risk map) calculation methods.

Population and environment safety regulation issue is without controversy a complicated scientific and technical task on transfer from technical to economic safety criteria (economic risk indicators).

It is logical to assume that cost components of safety provision program for objects with increased importance level should be stated as a separate item including resource verification methodology description. Above-mentioned requirements and normative assumptions should be considered during development of:

- COIIL technical maintenance programs;

- models for resource verification on responsibility, energy efficiency and safety level;

- structure systems for COIIL engineering system control and monitoring. 


\section{COIIL resource verification model development}

Verification is a type of check (test, research, study, and other) of product characteristics in compliance with specified requirements. Verification result is a conclusion of product compliance (or non-compliance).

It is obvious that core process approach theories based on anticipatory reality reflection [15-17] should be used for COIIL resource verification model development.

The verification model being developed reflects construction objects complexity. In theory, this is a system of function-compatible and interrelated complex elements, parameters of which interrelate on system reliability in general, considering values and indices random variation during the whole operation period.

Qualitative characteristics of the object element system is determined, recommended values of relative structure reliability $(y=1-\varepsilon)$ structure damage rate $\left(\varepsilon_{i}\right)$ coefficients are adjusted:

-«...standard-compliant technical condition is a technical condition category, when quantitative and qualitative parameter values of all criteria for technical condition assessment of building constructions and structures, including bottom soil condition, comply with values stated in design documents considering their variation ranges (GOST $31937-2011$ Technical condition evaluation and monitoring rules): $y=0,95 \ldots 1$ и $\varepsilon_{i}=0 \ldots 0,005$;

- constrained operational condition: $y=0,5 \ldots 0,84$ и $\varepsilon_{i}=0,16 \ldots 0,5$;

- emergency condition: $y=0,25 \ldots 0,49$ and $\varepsilon_{i}=0,51 \ldots 0,75$.

To substantiate the hypothesis on inadvertently stated complex interaction system "human - object - technology - equipment - environment" it is adopted that risk of functional parameters accidental variation under multicriteria factor interaction should be accessed in conjunction with forecast of various aspects of manmade environment development (technical, economical, psychological, social, and other).

In terms of quantity, risk value $\left(K_{p}\right)$ includes data on reliability $\left(k_{\mathrm{H}}\right)$, safety $\left(k_{\tilde{\sigma}}\right)$, ecological compatibility $\left(k_{\ni}\right)$, operation costs $\left(k_{3}\right)$, social phenomena $\left(k_{\mathrm{c}}\right)$, and other features, i.e.:

$$
K_{p}=k_{H} \cdot f(t)+k_{\sigma} \cdot f(t)+k_{\ni} \cdot f(t)+k_{3} \cdot f(t)+k_{c} \cdot f(t)+\ldots+k_{i} \cdot f(t)
$$

Presented studies are based on a hypothesis which emphasizes the safety level. This allows to consider other features as marginal ones [9, 12-17]. Safety parameter for a single COIIL element is specified with risk value $110^{-\mathrm{n}}$ for the whole resource period.

The developed verification model is represented by an alternative process algorithm for safety level smart control, which includes scientific and technical maintenance of risk situations rating and parameter normalization.

Every system state variation process over time corresponds to a certain state trajectory in space.

Every structural element of a building, and all built-in engineering systems and equipment have preset standardized reliability and resource parameters ensuring safe functioning of these elements and safe object operation in general. During resource period these parameters have variable nature characterized by the following expression $[1,15,17]$ :

$$
R_{t}=R_{t_{0}} e^{\alpha t},
$$

where $R_{t}$ - resource parameter actual value;

$R_{t 0}$ - parameter value which is conventionally taken as basic one;

$e$-base of the natural logarithms; 
$\alpha-$ resource parameter variation intensity;

$t$ - period since operation in compliance to $R_{t}$.

Resource parameter variation intensity is taken as a key calculation parameter, i.e. one can analyze shift value of security protection standardized indicator during operation period. According to ISO $5725.1 \mathrm{drift}$ is the difference between mathematical expectation of check results and adopted normal value.

It should be mentioned that operation period expansion results in hazard occurrence indicator shift towards severity increase

$$
\alpha=1-\eta \ln P,
$$

where $\eta$ - shift coefficient;

$P$ - system failure probability at the risk of hazardous situation occurrence.

It makes sense to qualify as high-risk scenario the situation, which results in variation of a personal self-fulfillment ability, i.e. harm to health which cannot be emotionally and/or physically compensated.

\section{Example of COIIL resource verification graphing}

The manufacturer assigns reliability indicator for every COIIL element. Indicator standardized values are set within the range of $80-100 \%$.

The aim is to determine probability of hazardous situation occurrence under varying reliability parameter shift during operation period.

By severity limit value variation (lower and upper limits) we obtained theoretical values of shift coefficient represented at graphical chart (fig.1). It provides visual assessment of indicator variation consequences, and weight arranging limits assignment for COIIL system elements in general.

Criterion evaluation was executed, range limits for shift level indicator were assigned: from 1 -operation start period, to 4,5 - factor influence corresponding to maximum resource.

Calculations assume that object reliability keeps at $80 \%$ level during the whole resource period, which is achieved by various management and maintenance activities.

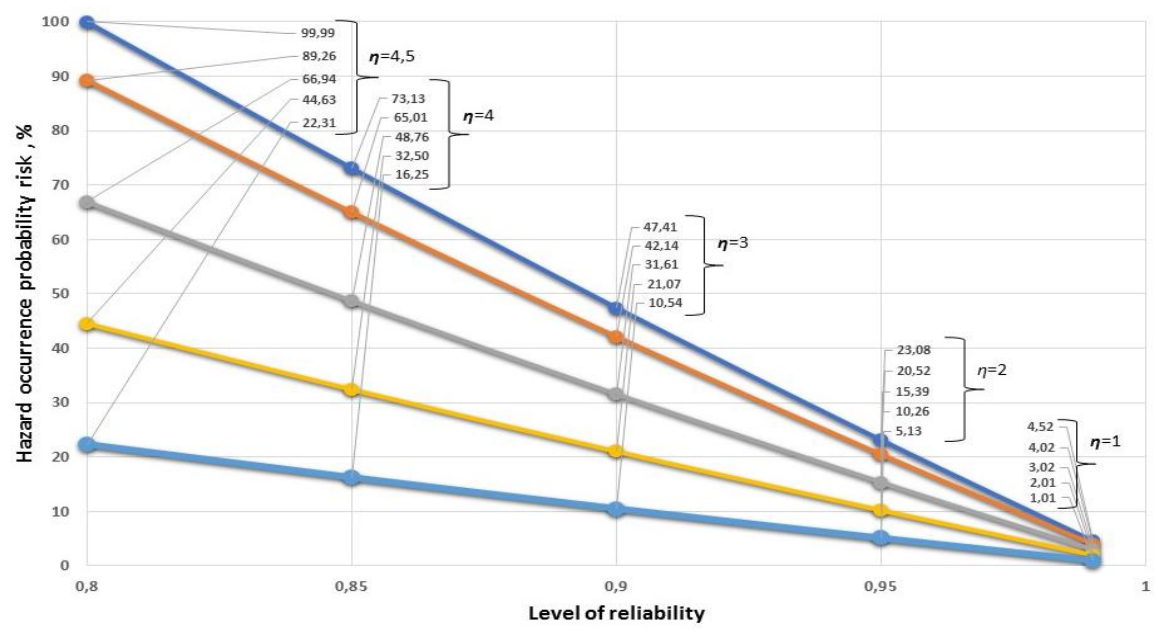

Fig. 1. Variation characteristic of interaction between COIIL reliability level indicator and hazard occurrence probability risk under various shift values 
Obtained graphs are linear due to theoretical parameter valuation for the purpose of problem solution and result visualization during developed method implementation. The graph illustrates that it is infeasible to reduce gamma-resource standardized by value: $80 \%$ reliability indicator. Critical level of shift value increase $-4,5$ - is also adjusted.

The proposed method of calculation visualization by graph allows to solve inverse problem: we can set restrictions on controlled parameter shift value for specified reliability level and set hazard occurrence probability.

\section{Prospects for implementation}

The developed method is taken as a basis for safety provisions rating by various criteria, in particular, by ecological risks with hazard level criterion introduction [18-23].

It is proposed to implement the developed method in addition to COIIL verification model during setting regulation reliability requirements.

\section{Conclusions}

1. Inconsistency in method standardizing and regulating building reliability during operation period with ultra-long resource period was found out.

2. It has been established that scientific notations in common take construction infrastructure as a complicated system capable for self-development and process selfregulation.

3. It is proposed to use variable verification modelling process approach for construction object reliability substantiation.

4. "Safety" indicator was accentuated as the principal indicator among other complex reliability indicators.

5. Verification model reflects complexity of construction objects.

6. Alternative algorithm for safety level smart control was presented. The algorithm includes scientific and technical rating of hazardous situations, and their parametric standardization.

7. Graphical visualization of hazardous situation occurrence risk probability under varying shift of reliability parameter during operation period was presented. The inverse problem was also solved: restrictions on controlled parameters shift values are introduced for specified reliability level and set hazard occurrence probability.

This study was supported by project of basic research to ensure the increased participation of subordinate educational organization in the implementation of the National technology initiative No. 13.11847.2018/11.12 "Development of a model of operational management of urban gas supply systems for basis, of the principle of regulatory perturbations", National Research Moscow State University of Civil Engineering, Moscow, Russia.

\section{References}

1. Yu. A. Verigin, The synergistic framework of processes and technologies: monograph (Barnaul: publishing house of Altai state technical University, 158, 2007)

2. E. Gusakova, Life cycle organization of the construction project (M: Fund "New Millennium",256, 2004)

3. V. Lebedev, M., A. A. Lebedev, Bull. of BSTU V. G. Shukhov, 3 (2010)

4. I. M. Smolyar, Architecture and city of Moscow, 2 (2000)

5. G. Shcherbina, Economic and legal issues, 12 (2010) 
6. E. Korol, P. Kagan, T. Barabanova, I. Bunkina, Intern. J. of Appl. Engin. Res., 1691-93, 11, 3 (2016)

7. E. A. Korol, P. B. Kagan, Intern. J. of Appl. Engin. Res., 1649-52,10, 23 (2015)

8. D. G. Dontsov, N. G. Yushkova, AIP Conf. Proc., III Intern. Young Res. Conf., 050011, (2017)

9. N. N. Nikonov, A. P. Melchakov, V. N. Rudin, J. "PGS", 3 (2013)

10. A. H. Baiburin, A. P. Melchakov, Arch., urban plan., des., 3-10, 11 (2017)

11. D. G. Dontsov, Bull. of VolgSUAC. Ser.: Constr. and arch., 38 (2014)

12. A. P. Malchikov, D. V., Cheboksarov, Prognosis, assessment and management of risk of accidents of buildings and constructions: theory, methodology and engineering applications: a monograph (Chelyabinsk: Publ. house of the SUSU, 113, 2009)

13. Y. I. Polyakov, J. of the res. center, 154-64, 2 (2008)

14. V. I. Telichenko, S. I. Zavalishin, M. S. Khlystunov, Sat. SPC "Urban construction complex and safety of life support of citizens". (M.: MSU, 211-18, (2005)

15. A A Bogomolov, M. V. Bunin, N. S. Sevryugina, Structure and semantics of variational optimization of transport machines and technological processes in the General theory of systems: monograph, (Belgorod: Publ. house of BSTU, 83, 2009)

16. A. V. Goropashnaya, St. Pet. University Vestnik, 19-32, 10, 1 (2009)

17. N. S. Sevryugina, Theory of formation of technical safety of the full life cycle of transport and technological machines: monograph (Belgorod: Publ. house of BSTU, 179, 2012)

18. N. Sevryugina, Theor. Foun. of Civil Engin. MATEC Web of Conf., XXVI R-S-P Seminar 2017, 00151, 117 (2017)

19. S. E.Grechishchev, M. A. Magomedgadzhieva, S. P. Dmitrieva, Proceedings of the Intern. Offshore and Polar Engin. Conf. ISOPE, Seoul, (2005)

20. V. Telichenko, I. Dorogan Intern. J. of Appl. Engin. Res., 11, 3 (2016)

21. A. B. Zhabin, A. D. Fomichev, Ju. N. Naumov, D. Ja. Solovyh Eurasian Mining, 1, 25 (2016)

22. P. Kapyrin, N. Sevryugina, IOP Conf. Ser.: Mater. Science and Engin., (2018)

23. N. Sevryugina, Adv. in Intel. Syst. and Comp., 692 (2018) 